\title{
Nucleolar behavior in pollen mother cells in the pigeonpea
}

\author{
P.S. Kumar \\ N. C. Subrahmanyam \\ D. G. Faris
}

The first two authors are affiliated with the Crop Genetics Laboratory, School of Life Sciences, University of Hyderabad, Hyderabad 5000134. India; the third author is principal pigeonpea treder, ICRISAT, Patancheru 502324, India. They thank Dr. J. P. Moss for providing the use of facilities at the Groundnut Cytogenetics Laboratory at.ICRISAT. (C) 1987, American Genetic Association.

\begin{abstract}
Reports on the number of satellite chromosomes in the karyotype of Cajanus cajan are controversial, with the number varying from zero to two. Expression of nucleolar organizer chromosomes during meiosis (prophase-l, telophase-l and telophase-lI) is used as an index for the number of nucleolar organizers and conclusive evidence is provided for the presence of two nucleolar organizer chromosomes in Cajanus and also its related species in the genus Atylosia. Nucleolar number in an autotetraploid of Cajanus confirmed the evidence from the diploid Cajanus for two nucleolar organizer chromosomes. Variation in the nucleolar size suggests that the two nucleolar organizer chromosomes differ in their activity.
\end{abstract}

PIGEONPEA [Cajanus cajan $(n=11 ; 2 n=$ 22)], an important pulse crop in the semiarid tropics, is not amenable to cytological studies because of its small chromosomes and the difficulty in obtaining good chromosome spreads. Nevertheless, there have been attempts to karyotype the somatic complement ${ }^{11,13-15}$, and of the pachytene complement $^{5,12}$. In addition to minor variations in chromosome lengths in different studies, the apparent number of satellite (SAT)/nucleolar organizing (NO) chromosomes were different in different studies. For instance, the studies of Shrivastava et al. ${ }^{14}$ and Sinha and Kumar $^{15}$ revealed one pair of SAT chromosomes in some cultivars of Cajanus cajan and none in others, those of Sharma and Gupta ${ }^{13}$ did not show any SAT chromosome, while studies of Pundir ${ }^{11}$. revealed two SAT pairs in pigeonpeas. The pachytene studies of Reddy ${ }^{12}$ and Dundas et al. ${ }^{5}$ indicate one pair of nucleolar organizers.

It has been clearly established that the maximum number of SAT chromosomes never exceed the maximum number of nucleolar organizers $8,16,17$. The present paper aims at resolving the discrepancies regarding the number of nucleolar organizers $(\mathrm{NO})$ in pigeonpea by examining the expression of NOs in pollen mother cells of Cajanus cajan and six related species in the genus Atylosia, which share a common chromosome number with Cajanus.

\section{Materials and Methods}

Eight diverse lines of C. cajan (Pant A2, C 11, Baigani, ICP 7035, ICPL 32, ICPL 47,
ICPL 59, and ICPL 95), a spontaneous tetraploid of Cajanus and six species of Atylosia (A. albicans, A. cajanifolia, A. grandifolia, $A$. lanceolata, $A$. lineata, and $A$. scarabaeoides) were used in this study. The tetraploid Cajanus cajan used in this study was isolated from an open population of Cajanus by Dr. K. B. Saxena, ICRISAT.

For meiotic studies, flower buds fixed in Carnoy's fluid were squashed in 1 percent acetocarmine followed by destaining with 45 percent acetic acid and restaining with 2 percent acetocarmine.

\section{Results and Discussion}

A maximum of two nucleoli were found in $<3$ percent PMCs at pachytene (Figure $1 A$ ) in Cajanus as well as Atylosia species. At diakinesis cells with two nucleoli also were occasionally observed (Figure $1 B$ ) but cells containing a single nucleolus associated with two bivalents (Figure $1 C$ and $D$ ) were common. The frequency of cells containing the maximum number of nucleoli depends on the rate of fusion and is different in the different species studied.

In Cajanus the number of nucleoli perPMC varied from 2 to 4 at telophase I. (Figure $1 E$ ) and 4 to 8 at telophase II (Figure 1 $F-H)$ irrespective of the cultivar used. Similar variations were evident in Atylosia grandifolia, A. lineata and $A$. albicans while in $A$. cajanifolia, $A$. lanceolata and $A$. scarabaeoides, the maximum number of nucleoli at telophase II was seven. The maximum : number of nucleoli in each daughter nucleus at telophase I or II, never exceeded two (Fig- 

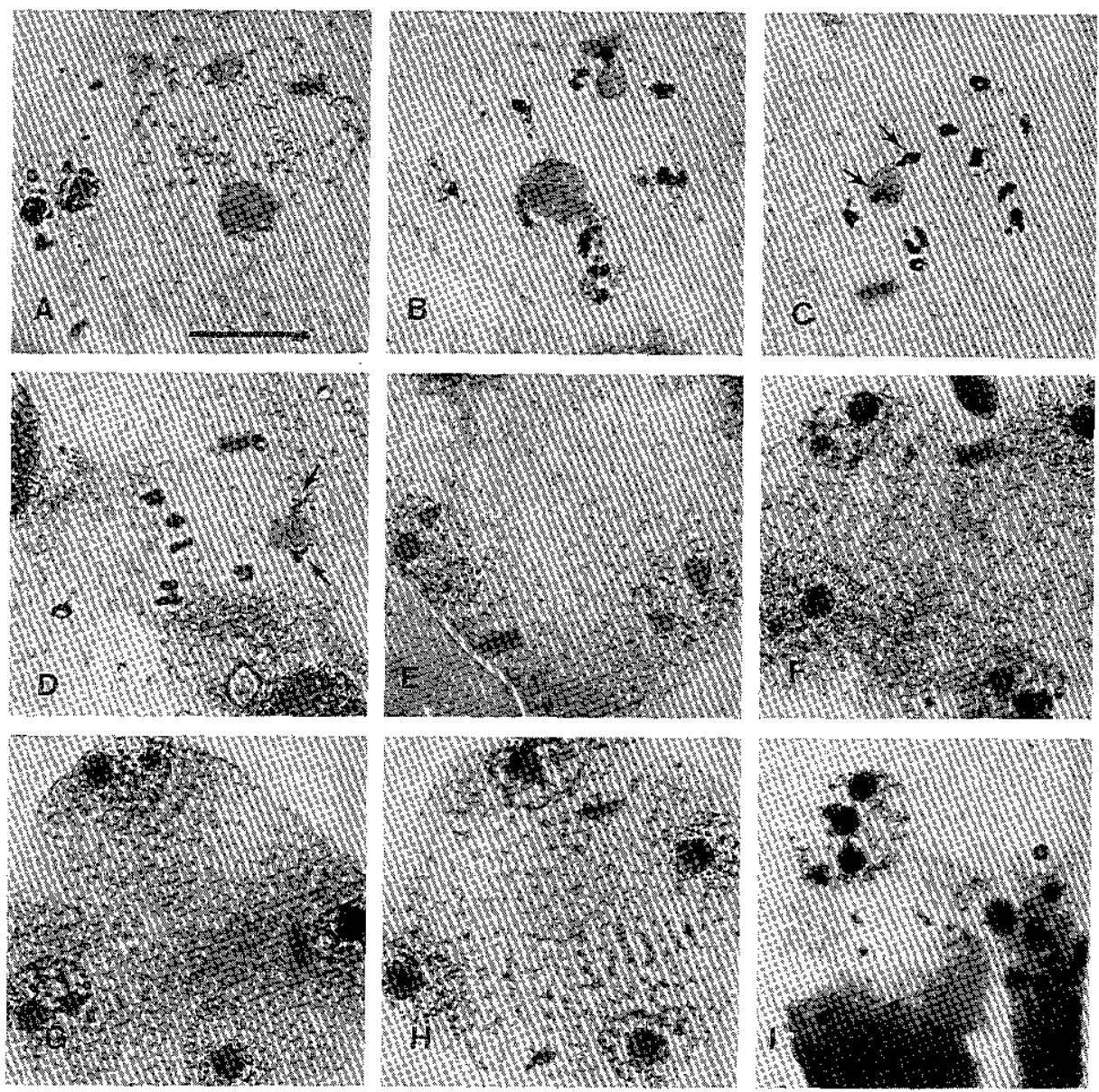

FIGURE 1 Nucleolar behavior in pollen mother cells of Cajanus cajan and Atylosia species. A-two nucleoli at pachytene in Cajanus cajan. (Bar indicates $10 \mu \mathrm{m}$.) B-two nucleoli at diakinesis in Atylosia lanceolata. C-diakinesis showing two bivalents (arrows) attached to the nucleolus in Cajanus cajan. $D$-diakinesis showing two bivalents (arrows) attached to the nucleolus in Atylosia cajanifolia. Etelophase I nucleolar distribution in Cajanus cajan: 2-2. F-H-telophase II nucleolar distribution in Cajanus cajan; $F: 2-2-2-2 ; G: 2-2-1-1 ; H: 1 \sim 1-1-1$. I-telophase I nucleolar distribution in tetraploid Cajanus cajan: $4-4$.

ure $1 E-H)$ irrespective of the cultivar or species studied, suggesting the presence of two nucleolar organizers per genome in pigeonpeas. Furthermore, the occurrence of a maximum of two nucleoli at prophase $I$ and the presence of nucleoli associated with two bivalents at diplotene/diakinesis also are indicative of two pairs of nucleolar organizers in the Cajanus and Atylosia species studied. Meiotic studies in the autotetraploid of $\mathrm{Ca}$ janus revealed a maximum of eight primary nucleoli at telophase I (Figure $i I$ ) substantiating the conclusions drawn from the studies on diploids.

The variations in the frequencies of PMCs at telophase II with different numbers of nucleoli in different species are presented in Figure 2. The variation in the frequencies of PMCs with different numbers of nucleoli (4 to 8 ) is a reflection of the nucleolar fusion that is very common in meiosis $3,4,6,18$. Invariably among the species studied the most frequent type was PMCs with four nucleoli, while the least frequent type had eight nucleoli. The preponderance of PMCs with four nucleoli (one nucleolus per daughter nucleus) at telophase II, is indicative of the fusion of two nucleoli in each daughter nucleus. However, it is evident from Figure 2 that in all the species with up to eight nucleoli per telophase II PMC the frequency of PMCs with at least one daughter nucleus possessing both the primary nucleoli was $>50$ percent, while in the other species, which had a maximum of seven nucleoli, the frequency of PMCs containing at least one daughter nucleus with two nucleoli was $<50$ percent, thereby indicating that nucleolar fusion pro-
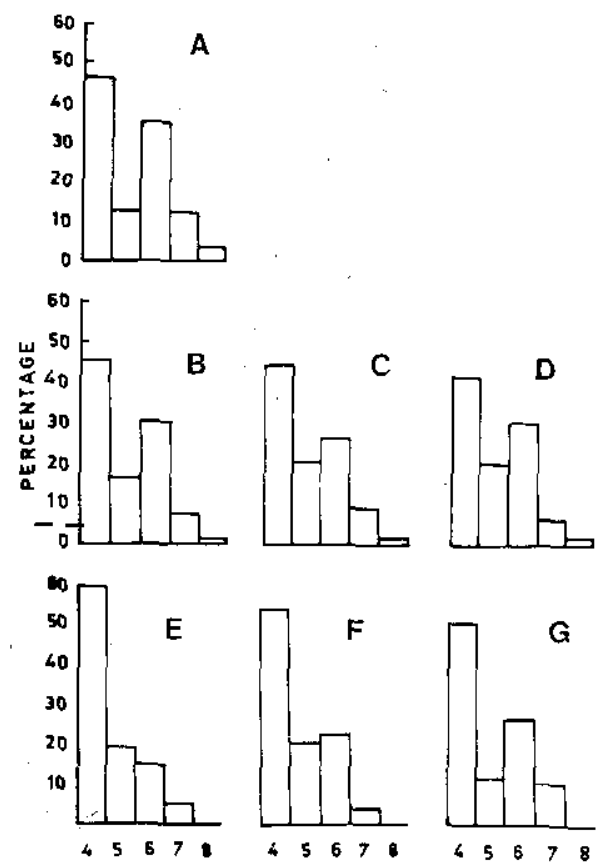

NO. OF NUCLEOLI

FIGURE 2 Frequency distribution of nucleoli at telophase II in pollen mother cells of Cajanus ca$j a n$ and Atylosia species. A-Cajanus cajan; $B-$ Atylosia grandifolia; $C$-Atylosia lineata; $D$ Atylosia albicans; $E$-Atylosia latisepala; $F-$ Atylosia cajanifolia; and $G-A t y l o s i a$ scarabaeoides.

ceeds at a relatively faster rate in $A$. latisepala, $A$. cajanifolia, and $A$. scarabaeoides than in the other species.

In the PMCs with two nucleoli per daughter nucleus, one nucleolus in each of the daughter nuclei was consistently larger than the other (Figure $1 A, B, F$, and $G$ ) in all the species in the present study. This would indicate that the two nucleolar organizers in each genome differ in their activity. Cultivated barley is a well known example for such differences $1,8,9,16$. According to Heiti ${ }^{7}$, the number of telophase nucleoli is constant, as well as the number of nucleolar organizers and SAT chromosomes for a given karyotype acquires a new meaning in the light of the evidence that genes coding for ribosomal RNA are located in the nucleolar organizer region (NOR) and the maximum number of nucleoli per nucleus corresponds to the number of rRNA synthesizing loci $1,6,8-10,16,17$. For the karyotype of the pigeonpea, there have been contradictory reports on the number of SAT chromosomes with Sinha and Kumar ${ }^{15}$ reporting lack of SAT chromosomes in certain cultivars of $C_{\text {: }}^{-}$cajan and Shrivastava et al. ${ }^{14}$ recording one SAT chromosome per genome in Cajanus while Pundir ${ }^{11}$ found two 
SAT chromosomes per genome in Cajanus and Atylosia species. Our data on the nucleolar behavior in PMCs conclusively establish two nucleolar organizers per genome in the Cajanus and Atylosia species studied. The differences in the size of the two nucleoli in daughter nuclei may reflect differences in the degree of the activity and/or differences in the multiplicity of $\mathrm{R}$ RA cistrons in the two nucleolar organizers in each species studied.

The discrepancies for the SAT-chromosome number in the karyotype of the pigeonpea could be a manifestation of the limitations in the technique employed for resolving the secondary constriction. There have been some studies where the controversies in nucleolar organizer number have been resolved using the mitotic nucleolar number as an index of NO activity ${ }^{2,8}$, but the present study has for the first time employed SAT-chromosome behavior during meiosis as an index of NO number.

\section{References}

1: Anastassova-Kristeva, M., H., Nico-
LOFF, G. KUNZEL, and R. RIEGER. Nucleolus formation in structurally reconstructed barley karyotypes with six satellite chromosomes. Chromosoma 62:111-119. 1977.

2. - - a , and S. GEORGIEv. Revision of SAT-chromosome number in Triticum monococcum with respect to nucleolar organizers activity. TAG 55:229-231. 1978.

3. BENNETT, M. D., M. K. RAO, J. B. SMITH, and M. B. BAYLISS. Cell development in the anther, the ovule and the young seed of Triticum aestivum L. var. Chinese Spring. Phil. Trans. R. Soc. Ser. B. 266:39-81. 1973.

4. DaRvey, N. L. and C. J. DRISCOLL. Nucleolar behaviour in Triticum. Chromosoma 36:131139. 1972.

5. DUNDAS, I. S., E. J. BRITTEN, and D. E. BYTH. Pachytene chromosome identification by a key based on chromomeres in the pigeonpea. $J$. Hered. 74:461-464. 1983.

6. Flavell, R. B. and M. O'Dell. The genetic control of nucleolus formation in wheat. Chromosoma $71: 135-152.1979$.

7. HeITZ, E. Die ursache der gesatzmassigen zaht, Lage-Form and Grosse pflanzlicher Nukleolen. Planta 12:775-884. 1931.

8. Jessop, C. M. and N. C. Subrahmanyam. Nucleolar number variation in Hordeum species and interspecific hybrids. Genetica 64:93-100. 1984.

9. LINDE-LAURSEN, I. Nucleolar organizer polymorphism in barley, Hordeum vulgare $\mathrm{L}$. Heridi tas 100:33-44. 1984.
10. NiCOLOFF, H., M. ANAST'ASSOVA-KRISTEVA, $M$. KUNZEL, and R. RIEGER. The behaviour of nucleolus organizers in structurally changed karyotypes of barley. Chromosoma 62:103-109. 1977.

11. PUNDrR, R. P. S. Relationships among Cajanus, Atylosia and Rhynchosia species. Ph.D. thesis, Banaras Hindu University, Varanasi, India. 1981.

12. REDDY, L. J. Pachytene analysis in Cajanus cajan, Atylosia lineata and their hybrid. Cytologia 46:397-412. 1981.

13. Sharma, P. C. and P. K. GuPTA. Karyotypes in some pulse crops. Nucleus 25:181-185. 1982.

14. ShrivastaVA, M.P., D. SHARMA, and SingH LAXMAN. Karyotype analysis in 15 varieties of Cajanus cajan (L.) Millsp. and Atylosia linea (W.\&A.). Cytologia 38:219-227. 1973.

15. SINHA, S. S. N. and P. KUMAR. Mitotic analysis in thirteen varieties of Cajanus cajan (L.) Millsp. Cytologia 44:571-580. 1979.

16. Subrahmanyam, N. C. and A. A. Azad. Trisomic analysis of ribosomal RNA cistron multiplicity in barley (Hordeum vulgare L.). Chromosoma 61:225-264. 1978.

17. — and - Nucleoli and ribosomal RNA cistron multiplicity in barley (Hordeum vulgare L.). Chromosoma 69:265-273. 1978.

18. SybeNGA, J. General Cytogenetics. North-Holland Publ. Co., Amsterdam. p. 158-159. 1972. 